\title{
Inbnltanerżeidnif.
}

Erfter thril.

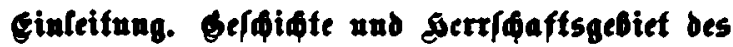
EReiфsprefgereques.

I. \$. 1. Das Brebredt. Begrif, Eintbeilung, Quellen, Eiteratur . . . . . . . . . 1

II. 8. 2. Die Entitebungägef́lidte Dez Reidz: preggejęes von 1874 . . . . . . . . 4

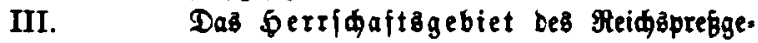
jepes.

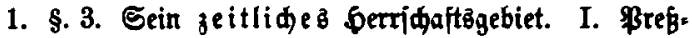
polizeired)t. II. Breßitrafredt. III. Berbot, Berjährung, Befdlagnahme . . . . .

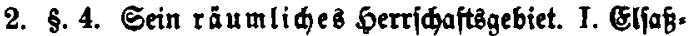
Eotbringen. II. \$reßsgewerbe, Breßpolizei, Prebftrafredt . . . . . . . . . . .

3. 5.5. Sein $\{a d l i \phi$ e Gerridaftżgebiet. Die

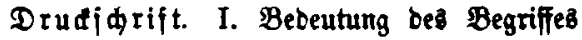
für daz ßreßredt. II. Die Druafdrift im engeren und weiteren Sinne. III. Sie mus zur Berbreitung beftimmt fein. IV. Dte Drud.

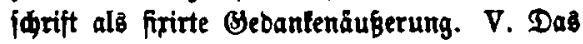
Subsgeben ber Druđjळrift als Begriffïmertmal

5. 6. Fortiepung. Die periobifíge Drudidrift. I. 3bre Bebeutury im Brébredte. II. Der Begriff. Eieferungabwerte unb Beilagen. III. Sonberredt ber periobifden Drudidrift. IV. Die autographirten Rorteşponbenzen 
4. §. 7. Daริ perīñ Seite

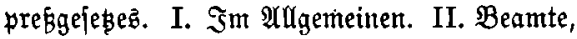
Militärperjonen u. $\lceil$. w. III. Befondere $\mathfrak{B} e=$ freiungen. IV. Die einer verjめäriten Berant= mortlidfeit unterworfenen ßerionen. . . .

§. 8. Fortieţung. 1. Der Dru a er. I. Definition.

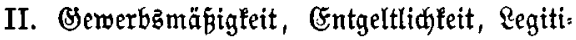

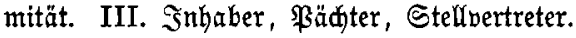
IV. Mehrere an Derjelben Druđfidrift bethei=

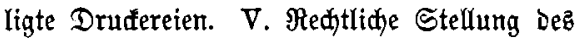
Drưterz. VI. Daz Befeb lennt feinen "ver= antwortliden “ Drufer . . . . . . . . 29

§. 9. Frortiebung. 2. Der $\mathfrak{B}$ erleger. I. Definition. II. Bemerbąmäßigfeit, (Entgeltlid)eit, Regiti: mität. Rommiffitonžperleger, Rommiffionär, Sortimenter. Selbjtwerlag. III. Sntaber, Bädter, Stellvertreter. Mebrere Berleger.

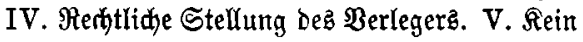
"Derantwortlidher" Berleger . . . . . .

§. 10. Fortiegung. 3. Der Berbreiter. Definition. Bemerbỉmäb̆tgleit H. f. m. Seine redtlidbe Stellung. 4. Der berauzgeber. Defintion. Redtllide Stellung . . . . . . . .

§. 11. Fortiesung. 5. Der $\Re$ ebafteur. I. Der $\mathfrak{B e}=$ griff im $\mathfrak{B r e \not b r e d t . ~ I I . ~ D e r ~ B e g r i f f ~ i m ~ R e b e n . ~}$ Rebafteur und Rebaftion. III. Der berant= wortlidie $\Re$ ebalteur. Mehrere verantw. $\Re e=$

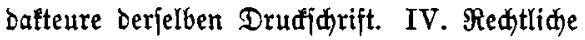

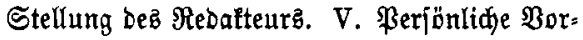
aubjęungen ber $\mathfrak{u}$ ebernahne ber verantmortl. Redaftion

5. §. 12. Suz̧p

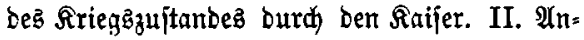
Dere $\mathfrak{F a ̈ l l e ~ b e r ~ S u ß p e n j i o n ~} . . . . . .$. 


\section{Das Erefgewerberedt.}

6. 13. Prebfreibeit uno Bemerbefreibeit. I. Definition Srite Der Brebgemerbe. II. Sie unterftehen ber Bes merbeorrmung. III. Gewerbefreibeit. IV. Beför: Derung von politifhen Beitungen; nidtgemerbz. mábige Berbreitung. V. Bewerbßmann, Stell. vertreter, ßäфter. VI. Bewerbeübertretungen. VII. neberfidt dea Stoffea. . . . . . .

5. 14. Der ftebende Betrieb ber Brebgewerbe. I. Defunition. II. Anzeige bes Betriebabeginues unb bes Rotales. III. Intauf von \$aren, Suden von Beftellungen. IV. Bewerbīmäbige iffentl. Berbreitung. V. Stellvertreter. VI. Entziebung ber Gemetbebefugniß . . . . . 47

5. 15. Betrieb ber Brebgemerbe in amber. zieben. I. Definition. Bebeuturtg. II. Regitimation§łфein. III. Stellbertreter. IV. Cntzie: hung ber Eemerbebefugnif . . . . . . 50

8. 16. Der fliegende Budhandel. I. Begriff und Bebeutung. II. Seine Formen: Solportage, Sammeln von श्Abontenten, gewerbämäbige, öffent. lide Berbreitung : - $\cdot \cdot \cdot \cdot \cdot \cdot \cdot \cdot \cdot \cdot$

8. 17. Das Platat. I. Seine gewerblide unb poli.

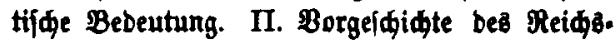
prébgefezes. III. Iragweite Des $\$ 30$ Xbf. 2

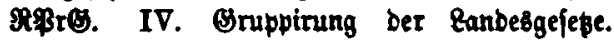

8. 18. Die burd bas Sogialiftengejeb gefídaf. fenen Befdränlungen ber Brésgewerbe. I. Heberfidt. II. Interjagung Des Gemerbebetriebes. III. Entzlebung ber Eewerbebefugnit. IV. Strafen. V. Betweigerung Des \&egititua tionbideines. VI. Der fleine BelagerungBzuftanb 
§. 19. Finanzielle Belaftungen der ßrȩ́ge= werbe. I. Allgemeines. II. Der Zeitungäjtempel unb bie Annoncenjteuer. III. Die Freieremplare

\section{Dritter Theil. \\ ærekpofizeiredf.}

§. 20. AIIgemeinez. I. Ueberjidyt. II. Exemptionen von ben prebpolizeilidyen Bejtimmunger. III. Jar

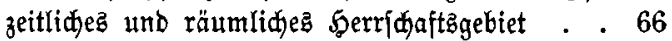

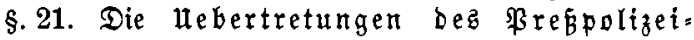
redites. I. Die Shuldlehre. II. Areib Der hafterben Berjonen. III. Bollendung und $\mathfrak{B e r}=$ jud Der ßreßppolizeidelitte. IV. Ronkurrenz V. Berjåhrung. VI. Rompetenz Der Beridite.

I. Die Rennung ber verantwortlidjen Berjonen auf ber Drudifrift.

§. 22. Die $\mathfrak{A n g}$ abe bez Druđerz unb $\mathfrak{B}$ erlegerz. I. 3weă ber Âtroronung. II. Snthalt ber $\mathfrak{A}$ in= gabe. III. Der Gewerbämann uno fein Stell= vertreter. Ingabe ber Firma. IV. Wohnont .

\$. 23. Die von biefer Angabe befreiten Dratz \{driften. I. Befeslidjer Umbreis ber Befret= ungen. II. Beitere Beifpiele. III. Albgrenzung. IV.-VII. Rajuijtil . . . . . . . . . 75

\$. 24. Die Rennung Dez verantwortliden Re= bafteura a uf periobifden Druafdrifter. I. Nentung Des Druđter unt Berlegerz.

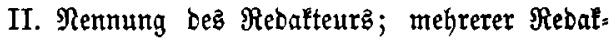
teure. III. Angabe des Mohnortes. IV. Ren= nung auf jeber Rummer . . . . . . . . . 78

8. 25. Die thebertretung biejer anorbnungen. I. Strafen. II. Buter Blaube. III. Âreiz ber Gaftenten ßerjonen. IV. Bollendung Deछ De= Iiftes. V. Fonturrenz . . . . . . . . . 82 
II. \$. 26. Die Pflidtezemplare. I. Shre Bebeutung.

II. Die Ler periobifden \$reffe obliegente Ber. pflidtung. III. Befreite Blätter. IV. Hebertre. tung unb Strafe. . . . . . . . .

II. \$. 27. Die Betpflidtung zur 9ufuabme von amtliden Betanutmodungen. I. Begrünbung tiefer Pflidt. II. Sie obliegt einem Thelle ber periobijden Prefle. III. 2xmtlide Betaut. madungen. IV. (Entridtumg ber Gimuidungz: gebübren. V. श्abbrud. VI. Uebertretung unb Etrafe. . . . . . . . . . . . .

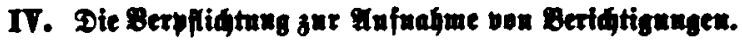

5. 28. Das Entgegnungsredt. I. Seine Ratur unt Bebeutung. II. Beftebt mur gegenúber ber periobifigen Preffe. III. Pitgetbetlte Iyatfadien. IV. Betbeiligte Berfonen. V. Begfall des Cntgegnumgsededte. VI. \$eitererzäblung von ân. Deten behaupteter Thatfaden . . . . . .

8. 29. Die sufnahmepflidt. I. Sie obliegt bem verantwortliøen Rebalteur. II. Sart Des 26. orndes. III. Gaftung für ben 3nbalt . . .

5. 30. Daż Beridtigung gैverfabren. I. Die ver. fđiebenen Syfteme. II. Das \$reidsprefgeles.

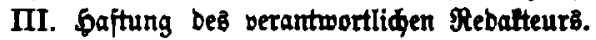
IV. Intrag exforberlid. V. Das Berfabren felbjt. VI. Ermeuerte \$eigerung. VII. BoL. enbung Des Delittes . . . . . . . . . 101

T. Das Berbot bet wetteren Bextrettung.

8. 31. Da Berbot ausănbifder Druajurif:

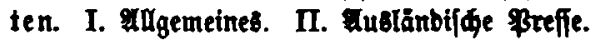

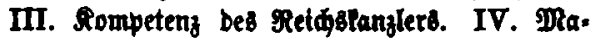
terielle Borausfesungen unb $\nabla$. zeitlide Be. grengung bes Fusiprudbredtes. VI. Tragmeite Des Berbotes. VII. Entzitehung bes \$softbebtts 
5. 32. Die uebertetung biejes Berbotes. Eeite I. Etraje. II. Renntuif Des Berbotes erforier,

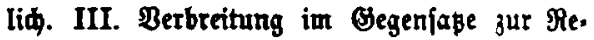
prosuttion. IV. SDentität ber verbetenen und Der verbreiteten Druxidurift.

8. 33. Das Berbot fozialbemolratifier Irud :

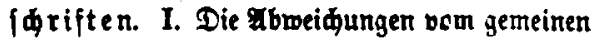
Redt. II. Sozialbemot. Sdriften. III. 3uftän= bigfeit. IV. Befantmadung an Berleger u. f. wo. V. Tragweite be Berbotes. VI. Berbreitung, Fortiegung, Mieberabbrud. VII. Etraje .

I. Die Beidlaguahme van Druffidriften.

8. 34. Strafprozeforonung und Pré̈gejep. I. Bebeutung ber Bejdlagnabme. II. Tie $\mathfrak{B}_{\ell}$. ftimmungen Der StrßrD. III. De lege fe-

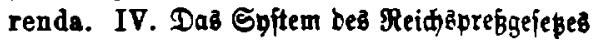

\$35. Die nidtríterlide Befdlagnabme in Breвladen. I. Boraublebunger. II. und III. Eine ftrafbare Gandlung wuf wenigitens

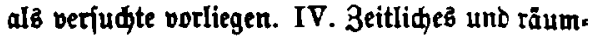
lides berridaftsgebiet biefer Bejtimmungen .

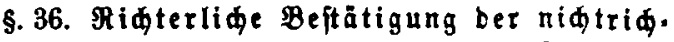
terliden Befdlagnahme. T. Rompetenz: bejtimmung. II. Stellung tea Geridtes ber Anflage gegenúber. III. Dą Beftätigungäver. fabren. IV. Medtemittel. $\nabla$. Einleitung ber Berfolgung. VI. Definitive Erledigung bes Be:

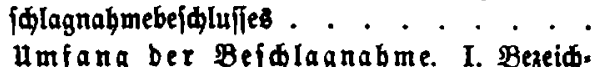
125

5. 37. Umfang ber Befdlagnabme. I. Bezeid: Gejepes. II. Trennbare Theile ber Drudifitif. III. Blatten unb formen. IV unb V. rie ber Bejdlagnabme unterliegenten Eremplare

§. 38. Wirfung Der BeidIagnahme. I. Berbrei, 
tung unb Bieberabtrud. II. Dauer bes $\mathfrak{B e t}$ : botes. III. Strafe ter uebertretung. IV. Das neben eventuell Saftung für ben Inbalt . .

8. 39. Die Befdlagnabme nad bem Sozias Iiftengejeß. I. Bejథlagnabme vetbotener, unb II. now nidt verbotener Drudidir. III. Ueber: tretungen

\section{vienter Utyeth. Ereffrafrest.}

I. Das Prefipelitt.

5. 40. Der Begriff bes Brebbelittes. I. Stant Det Anifiten. II. Prébelift und \$reßpolizeibelitt. III. Das Brebbelitt als normmibrige ftrafbate Gebantenäuberung, begangen bur屯 Berbreitung von Druthmriften. . . . . . 136

5. 41. Fortiebung. Confequenzen aus bem Bes griffe. I. Das Berbreiten ber Drutifdrift als Begeben bes Brebbelittes. II. Die weitere Berbreitung alb jelbftänbiges Delitt. III. Bollen: bung und Berfuch Der Prefbelitte. IV. Ron. hurtenz. Anhang. Rompeteng ber (S)eridte.

8. 42. Der Begriff Der Berbreitung. I. Seine Bebeutung im Prepredte. II. \$. 3 Rßre. III. Defintition. IV. unteridieb von bem भus: geben ber Dradidgrift. V. Berbreitung ift Bugäng. liфmaぬen und niळt 3ugăngliøjein. VI. Ber. breitung eines Exemplars. VII. Bublitum unb geidilofiener Arets. VIII. Der Sötper ber Drud. \{drift unb ibs 3nbalt. IX. Borbereitung unb unterftípung ber - Berbreitung . . . . . . 149

8. 43. Die prebredtlide Bebeutung ber Re: probution oon Drudidriften. I. Be. 
genfaß zur Berbreitung. II. Suriftijфe Ron. Eeite ftrultion. UI. Cie lann alz jelbjtänciges ßref. belift erideinen. IV. Rorfequenzen .... 155

\$. 44. Beridite über Beriftzoerbantungen. I. Suriftifige Roritrultion. II. Deffentlide Berbandlungen. III. Berbantlungen bei ge:

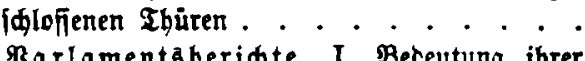

\$. 45. Barlamentaberidte. I. Beteutung ibrer Eonverftellung. II. Die Beridterftattung ift nidht nur niфht jtafbar fonterm aud nidt norm. mirrig. III. Wabrbeitęgetreue Beridte. Deffent. lidpe Eipungen . . . . . . . . . .

8. 46. Die im Prégejeße jelbit entbaltenen Brebielitte. I. Innere Natur ierfelben. II. Berbot Ier Berōffentlidung von Truppens bemegungen u. j. m. III. Deffentlide Iuffot. serung zum Aufbringen von Belcjtrajen u. f. w. IV. Beröffentlidung von EtrajprezeB : ङqrift. ftüđen .

II. Die Berantwortlidfeit fár \$reß̧belilte.

9. 47. Die veridiebenen Berantwortlidleits. injteme. I. Unzulänglidłeit ber allgemein= ftrajredtliden Brundfäpe. II. Dab belgifde Eyjtem. III. Die Fabrläfígteiţjftrajen. IV. Der Redalteur alż bolojer Thäter . . . . .

\$. 48. Die ReidBpreBgejeßgebung. I. Borge-

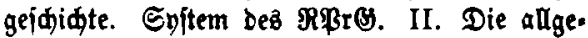
mein = frrafredtliden Gruntjäßze als Bajiz. III. Ergänzung Derfelben Durd die Quftung

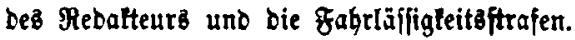

IV. Gerridaftägebiet biejer Bejtimmungen . . 166

Der verantmortlide $\mathfrak{R}$ edalteur alв $\mathfrak{T h a ̈ s}$ ter. I. Der verantwortlide \$etafteur. II. Die Präjuurption jeiner şăterfdajt. III. Das 


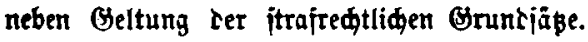

Grite

IV. Eandestredtlide Alnorbnungen. Anbang. Zeugnifzzmang gegen ren Resalteur . . . . 183

5. 50. Fortießung. Rajuiftit. I. Segenbeweia

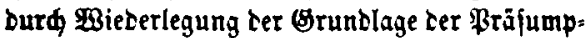
tion. II. Unjdultebeweis. III. Plangelnte Redtswistigleit

5.51. Die prebredtlide Fabrläffigteit. I. Âreis ter hajtenben ßerjonen. II. ßrājump: tion ber fabrlajijigfeit. III. Begenbemeis . .

8.52. Fortfeßung. Folgerungen aub ber iu. riftijen Ratur ber prébredtiden $\mathfrak{F a h r l a ̈ j f i g l e i t . ~ I . ~ E a d e m ~ c a u s a , ~ e x c e p t i o ~}$ rei judicatae. Antragabelitte, Bribattlage.

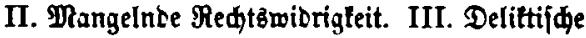
Thätigleit ijt aud bier baz Berbreiten. IV. Etrafe. V. Berjăhrung

III. §. 53. Die Berjährung ber Brefibelilte. I. Stand Der Gejeßggebung. II. Brünte für bie hürzere Berjāhnungäirifit. III. Berjolgungżverjâhrung. Breǵzelitte unt Préppolizeitelitte. IV. Beginn ber Frift. $\nabla$. unterbredung ter Berjäbrung .

\section{Die Unbraudbarmadung von Drudfidrifter.}

5. 54. Borausjeßungen ihrer 3uläjigleit. I. Dbjeltive Beteutung I. Druđjdrift. II. \$. 41 RStr(GB. III. Unteridied zmijden Beidlag. nahme und unbraudbarmadjung. IV. und $\nabla$. Der $\mathfrak{s}$ nhalt ber Drudjdrift mus normmibrig und frafbar fein. VI. Anmenbbarteit bez in.

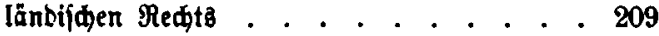

5. 55. Fortiebung. Die objeltive Strafbar:

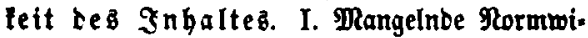
Drigleit. \$arlamentaberidjte. II. Die Strafbar. leit ber Ganblung im $\mathfrak{a n t e r i d i e b e ~ v o n ~ D e r ~}$ 
Durdfübrbarteit ber Straftlage und ber Be. Exite ftrafbarteit bes Zhäters. III. 3uläfifigleit ob. jettiver Mabregeln bei Intragsbelitten; bei ein. getretentr Beriābrung unb Begnarigung . . 215

5.56. $\mathfrak{u}$ mang und Birfung ber riditerlid angeorbneten anbraudbarmadung. I. Subbebnung auf \$latten unb formen. II. Be. jaränlung auf bie frafbaten Theile ver Drud. iqrift. III. Die ser Pabregel unterliegenden Exemplare

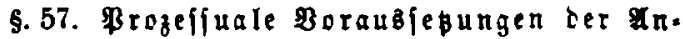
ortrung. I. Berbintung berfelben mit bem in ver פauptiade gefällten urtbeile. II. Das bejontere objettive Berfabren . . . . . 22 